\title{
Can Large Hydatid Cysts Resolve with Medical Treatment Alone?
}

\author{
Donald Simon Coenraad F.N. Koegelenberg Soumitra Sinha Roy \\ Brian W. Allwood Elvis M. Irusen \\ Division of Pulmonology, Department of Medicine, Stellenbosch University and Tygerberg Academic Hospital, \\ Cape Town, South Africa
}

\section{Established Facts}

- Data to support the sole use of albendazole for the cure of pulmonary echinococcal disease is lacking, apart from its use for patients who have refused surgery or in whom surgery is contraindicated.

\section{Novel Insights}

- Albendazole as primary treatment for multiple and large pulmonary echinococcal cysts can lead to cure.

\section{Key Words}

Hydatid · Echinococcal cysts · Benzimidazoles

\begin{abstract}
A 66-year-old female from a rural area in South Africa presented with non-life-threatening haemoptysis. Radiologic and serological investigations attributed her symptoms to bilateral, large echinococcal cysts. She declined surgery despite her lung physiologic parameters, which deemed her eligible. Medical therapy with oral albendazole was initiated with excellent clinical and radiologic response during a follow-up period of 18 months. To our knowledge, this is one of the first reported cases in the literature that shows complete resolution of bilateral large echinococcal cysts with medical treatment alone in an adult patient.
\end{abstract}

(c) 2016 S. Karger AG, Basel (c) 2016 S. Karger AG, Basel

0025-7931/16/0926-0428\$39.50/0

\section{Introduction}

Data for the use of primary medical treatment for the cure of large pulmonary echinococcal cysts is lacking. Medical management for pulmonary echinococcal cysts is reserved for poor surgical candidates, disseminated disease, and for those with intraoperative complications such as intraoperative spillage of hydatid fluid.

Hydatid disease or echinococcosis is caused by larvae of the tapeworm Echinococcus. Although four Echinococcal species are recognized, the vast majority of infestations in humans are caused by E. granulosus [1]. E. granulosus causes cystic echinococcosis, which has a worldwide distribution and is concentrated in sheep-raising areas. E. multilocularis, E. vogeli and E. oligarthrus constitute the other species, and are rare in South Africa. The liver and lungs are the most frequently involved organs

\section{KARGER}

E-Mail karger@karger.com www.karger.com/res
Dr. Donald Simon

Division of Pulmonology, Department of Medicine Stellenbosch University and Tygerberg Academic Hospital PO Box 241, Cape Town 8000 (South Africa)

E-Mail siya_bonga@icloud.com 

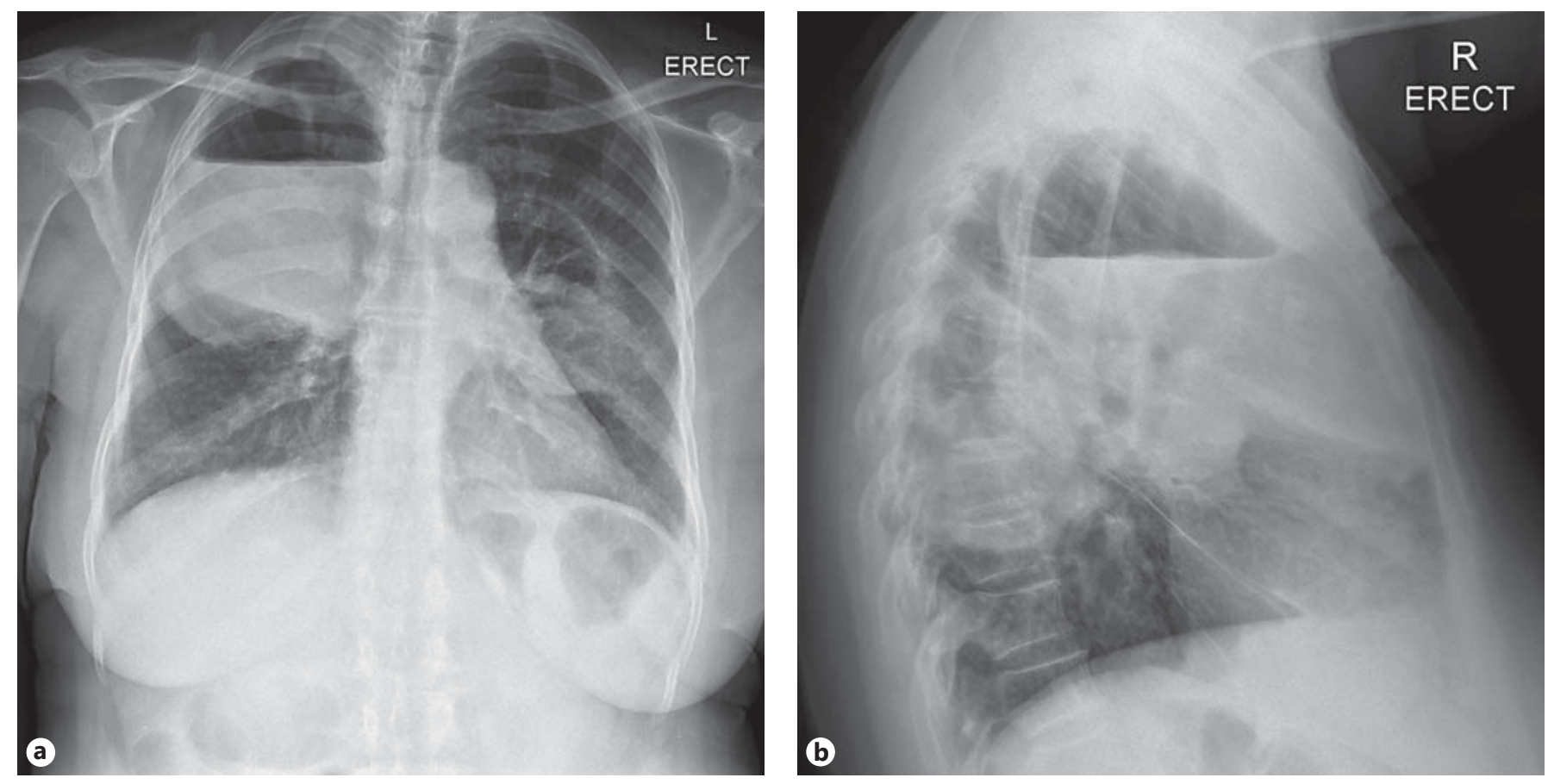

Fig. 1. a, b The patient's initial chest radiograph showing a right upper lobe and a right lower lobe hydatid cysts.

[1-4], and most patients with pulmonary echinococcosis are asymptomatic, while some may present with symptoms due to compressive effects of a large cyst, invasion of mediastinal structures, cyst rupture, and superinfection manifesting as a lung abscess [5]. Compression of adjacent structures can lead to dyspnoea, while erosion into adjacent structures causes bone pain, haemorrhage, or airflow limitation. The main complication is cyst rupture that may lead to cyst material flowing either into the bronchial tree producing cough, chest pain, haemoptysis, or a lung abscess; or into the pleural cavity, causing simple or tension pneumothorax, pleural effusion, or empyema $[5,6]$. Release of antigenic material and immunological reactions may lead to fever and acute hypersensitivity reactions ranging from urticaria and wheezing to lifethreatening anaphylaxis. Ruptured cyst membranes may also be evacuated entirely through the bronchi or can be retained to serve as a nidus for bacterial or fungal infection. Dissemination of protoscolices can result in multiple secondary echinococcal disease.

The diagnosis is usually made with a combination of imaging and serological testing. Surgery is considered the treatment of choice for those who are deemed fit to undergo it, because it can lead to cure by completely removing the parasite. The aim of surgery is to safely remove the parasite, prevent intraoperative dissemination, and to treat the peribronchial cyst. Medical treatment alone can be considered in patients with small cysts and in patients with a high surgical risk. Here, we report a case with multiple pulmonary echinococcal cysts with major radiological response to medical treatment alone.

\section{Case Report}

A 66-year-old female from a rural area in South Africa was referred to our unit for further evaluation of an apparent lung mass that was seen on a chest radiograph in July 2015. She gave a history of non-life-threatening recurrent haemoptysis and occasional night sweats, but denied any chest pain or loss of weight. She had no previous medical illnesses and she has never smoked, although reported biomass fuel exposure.

On clinical examination, she appeared well with a respiratory examination that was essentially normal. The rest of the physical examination was unremarkable.

Her spirometry was normal with a forced expired volume in $1 \mathrm{~s}\left(\mathrm{FEV}_{1}\right)$ of $83 \%$ of predicted and a forced vital capacity (FVC) of $97 \%$ of predicted. Her diffusion capacity for carbon monoxide $\left(\mathrm{DL}_{\mathrm{CO}}\right)$ was $58 \%$ predicted and her maximal oxygen consumption $\left(\mathrm{V}_{\mathrm{O} 2} \mathrm{max}\right)$ was $21 \mathrm{ml} / \mathrm{min} / \mathrm{kg}$, indicating that she is an eligible candidate for surgery.

Sputum microbiologic examinations were negative for mycobacterial and other bacterial pathogens. The patient's chest radio- 

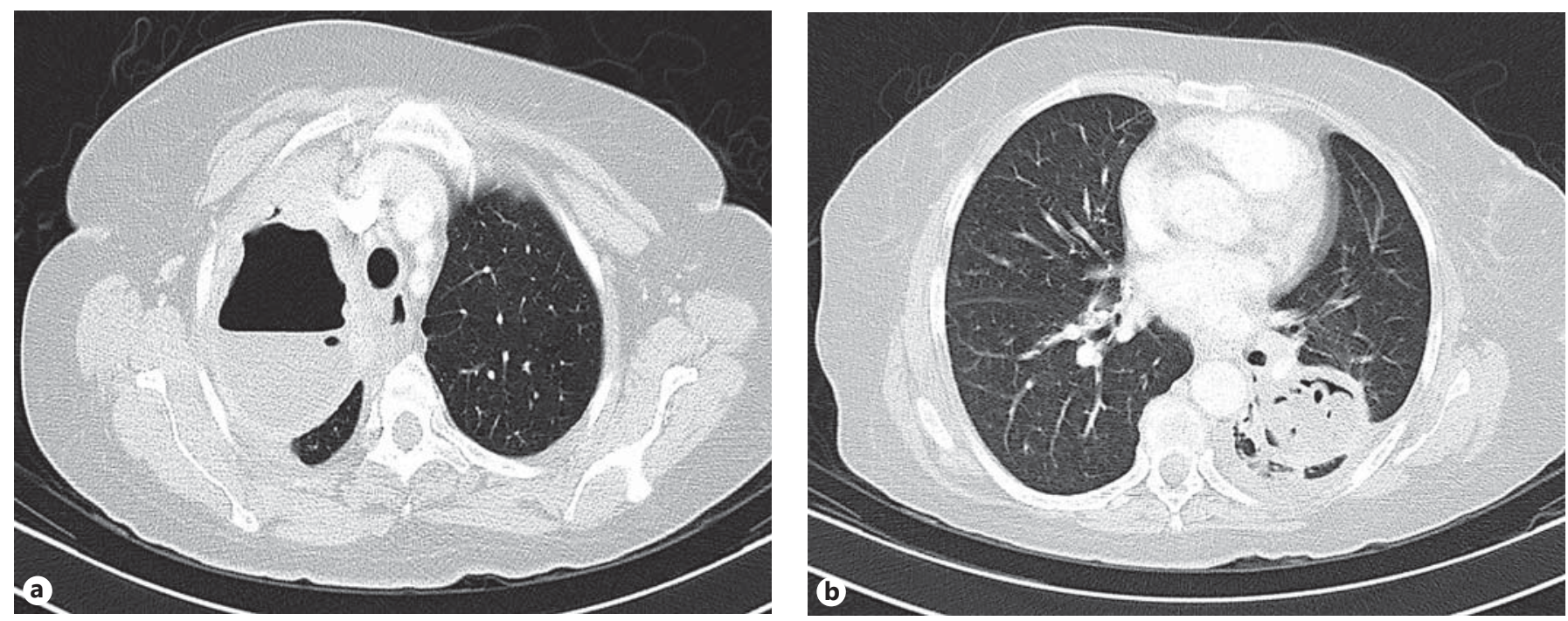

Fig. 2. a, b Chest computed tomography showing hydatid cysts at initial presentation.

graph (fig. 1a) revealed a large right upper zone opacity with an air-fluid level as well as a well-circumscribed homogenous opacity in the left lower lobe. These complicated cysts were confirmed on chest-computerised tomography which showed air-fluid levels and air inclusions (fig. 2a, b). No cysts were observed in her other organs. Serum E. granulosum enzyme immunoassay was strongly positive with a ratio of 10.5 [normal range: $0-1.1$, Ridascreen Echinococcal IgG (ELISA); R-Biopharm AG, Germany].

She was advised that surgical resection and removal of intact cysts would be potentially curative and the treatment of choice. In addition, she was assessed as having sufficient pulmonary reserve and deemed to be a good candidate for lung resection; however, she declined surgery for personal reasons. She was subsequently initiated on an uninterrupted course of albendazole $400 \mathrm{mg}$ twice daily and was followed up at 3-month intervals. After 6 months of treatment, her presenting symptoms had resolved, while her pulmonary function tests remained unchanged during the whole follow-up period. Serial chest radiography showed decreasing size of the cysts, and after 18 months there was almost complete resolution of both lesions (fig. 3). She has been given ongoing albendazole pending the 4-month examination. No complications attributable to albendazole were observed during treatment.

\section{Discussion}

Our case demonstrates that there may be a place for benzimidazoles in the management of large pulmonary cystic echinococcosis. Our patient, treated only with albendazole, had large bilateral echinococcal cysts, and with good pulmonary physiology would normally have been considered for surgical intervention, the recom-

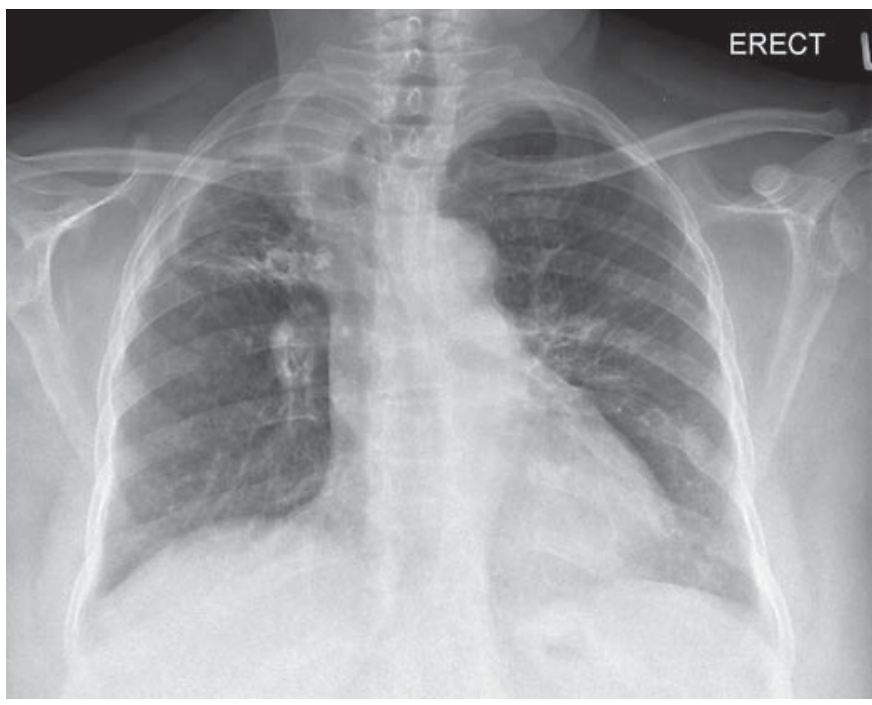

Fig. 3. The patient's chest radiograph following 18 months of albendazole therapy.

mended treatment of choice. For hepatic echinococcal cyst, other treatment options exist, such as the puncture, aspiration or injection of a helminthicide and reaspiration (PAIR) technique. However, for pulmonary echinococcal cysts, this technique resulted in more complications, is rarely indicated $[7,8]$, and alternative options are limited. Medical therapy with benzimidazoles is thought 
valuable in disseminated disease, including secondary lung or pleural involvement, poor surgical risk patients and when there is intraoperative spillage of hydatid fluid [9]. Further, medical treatment alone has been suggested by some for small pulmonary hydatid cysts $(<7 \mathrm{~mm}$ diameter) [10]. However, for large and multiple pulmonary echinococcosis, case reports on the successful medical management are lacking, and surgery remains the primary treatment and only hope for cure. In addition, prolonged medical treatment carries the risk of medication side effects and progression of disease in the case of large, multiple cysts.

Perhaps fortuitously, our patient opted to pursue medical management with a subsequent excellent response. The extent of her response was surprising, given current guidelines and literature that does not support primary medical treatment for large and multiple pulmonary hydatid disease. Traditionally, complicated, multiple, multiorgan, large, multicompartment cysts with thick or calcified surrounding adventitial reactions were thought relatively refractory to medical management and require surgical excision [10-12], although small, isolated cysts have occasionally been shown to completely respond.

Our case is unique in that our patient had large, bilateral echinococcal cysts that resolved on medical treatment. There is a lack of similar case reports describing cure in such adult patients, although one recent paediatric case from India describes the resolution of multiple bilateral large echinococcal cysts in a 12-year-old child following 6 months of treatment with albendazole [13]. In the developing world, pulmonary echinococcal disease is common, yet there is a lack of literature describing management in this setting. In a resource-constrained setting, cardiothoracic services are limited, and alternative evidence-based options to surgery may prove valuable.

In our patient, we are unsure of the optimum duration of therapy, and whether the response following cessation of medication will be maintained or whether the cysts will recur. Questions relating to duration of treatment, frequency of follow-up and patient selection remain unanswered. Case reports similar to ours may pave the way for comparative studies that should seek to answer these questions.

In conclusion, we have demonstrated the case of a patient with multiple and large pulmonary echinococcal cysts that was treated medically. This is one of the first reports of a curative response to medical treatment alone in an adult patient. Surgical resection remains the current treatment of choice for the management of large echinococcal cysts, unless data regarding the safety and efficacy of oral treatment becomes available.

\section{Financial Disclosure and Conflicts of Interest}

The authors have no conflicts of interest to disclose.

\section{References}

1 Schantz P: Echinococcosis; in Guerrant R, Walker DH, Weller PF (eds): Tropical Infectious Diseases: Principles, Pathogens and Practice. Philadelphia, WB Saunders, 1999, pp 1005-1025.

2 King CH: Cestodes (tapeworms); in Mandell GL, Bennett JE, Dolin R (eds): Principles and Practice of Infectious Diseases. New York, Churchill Livingstone, 1995, pp 2544-2553.

-3 Ammann RW, Eckert J: Cestodes. Echinococcus. Gastroenterol Clin North Am 1996;25: 655-689.

4 Kammerer WS, Schantz PM: Echinococcal disease. Infect Dis Clin North Am 1993;7: 605-618.

5 Morar R, Feldman C: Pulmonary echinococcosis. Eur Respir J 2003;21:1069-1077.
Santivanez S, Garcici HH: Pulmonary cystic echinococcosis. Curr Opin Pulm Med 2010; 16:257-261.

7 Filice C, Brunetti E: Use of PAIR in human cystic echinococcosis. Acta Trop 1997;64:95107.

-8 Akhan O, Ozmen MN, Dincer A, Gocmen A, Kalyoncu F: Percutaneous treatment of pulmonary hydatid cysts. Cardiovasc Intervent Radiol 1994;17:271-275.

-9 Kilani T, El Hammami S: Pulmonary hydatid and other lung parasitic infections. Curr Opin Pulm Med 2002;8:218-223.

10 Moro P, Schantz PM: Echinococcosis: A review. Int J Infect Dis 2009;13:125-133.
Bartoloni C, Tricerri A, Guidi L, Gambassi G: The efficacy of chemotherapy with mebendazole in human cystic echinococcosis: longterm follow-up of 52 patients. Ann Trop Med Parasitol 1992;86:249-256.

12 Galanakis E, Besis S, Pappa C, Nicolopoulos P, Lapatsanis P: Treatment of complicated pulmonary echinococcosis with albendazole in childhood. Scand J Infect Dis 1997;29:638640.

13 Siddiqui Z, Faridi SH, Siddiqui B, Singh KD: Complete resolution of bilateral pulmonary hydatid cysts with prolonged administration of albendazole: a rare occurrence. Int Arch BioMed Clin Res 2016;2:36-39. 